\title{
Imaging Shape Dependent Surface Plasmon Modes in Noble Metal Nanoparticles.
}

\author{
S. Aloni, Y. Yin, and A.P. Alivisatos \\ * The Molecular Foundry, Lawrence Berkeley National Laboratory, Berkeley, CA 94706
}

Nobel metal nanostructures are of great interest because of their unique optical properties. Their optical properties are determined by the surface plasmon resonance of conduction electrons, the frequency of which is determined not only by the nature of the metal or alloy of which the particle is made but also by the particle's size and shape. Moreover, the properties can be further tailored by forming nanoparticle assemblies and by controlling the surrounding dielectric medium [1]. For many metals the plasma frequency lies in the UV part of the spectrum and the nanoparticles do not display strong color effects. In noble metals, due to strong d-d band transitions, the plasma frequency is pushed into the visible part of the spectrum. Hence most of the surface plasmon experiments were carried out with gold silver and copper nanoparticles.

In this work we focus on study of the shape effects of the plasmonic excitations in silver and gold nanostructures. The silver and gold nanostructures were synthesized by solution phase synthesis [2] yielding highly faceted nanocrystals including spheres, cubes, triangular plates, bi-pyramids and rods of aspect ratios up to 1:15. The nanoparticle solutions were washed off the access surfactant and suspended on commercially available carbon and $\mathrm{SiOx}$ and $\mathrm{SiN}$ support grids for analysis.

Due their small size, the correlation of the shape and optical properties of individual nanocrystals is not straightforward.. Usually the geometrical shape is deduced from high-resolution TEM images while optical properties are measured via optical spectroscopy, which provides average information of large number of nanoparticles with varying sizes and shapes. We used high energy-resolution EELS spectral imaging in combination with Z-contrast imaging that was carried out using a FEI Tecnai F20 UT microscope operated at $200 \mathrm{kV}$. The microscope is equipped with a doublefocusing Wien filter acting as a monochromator directly below the field-emission gun. This setup in combination with a high-resolution Gatan Imaging Filter (GIF) and an improved highvoltage supply allows for a $2 \mathrm{~nm}$ sized electron probe an energy resolution of $0.25 \mathrm{eV}$ or better, thus energy-loss information can unambiguously be accessed down to about $1 \mathrm{eV}$ electron energy loss.

In a small spherical silver nanoparticle the optical response is defined by the dipole resonance mode at $3.1 \mathrm{eV}(395 \mathrm{~nm})$. This mode is size dependent exhibiting significant red-shift and broadening as the size increases. The contributions of the higher order modes, mainly quadrupole mode, dominate at sizes above $40 \mathrm{~nm}$ and are weakly size dependent. Figure 1c shows EEL spectra extracted from different positions in the spectral image of single spherical nanoparticle. When pointing the electron beam to the center of the nanoparticle the most significant energy loss mode can be observed at $\sim 3.8 \mathrm{eV}$, which was observed in silver thin films and is attributed to the bulk silver plasmon. By pointing at the particles edge we increase our sensitivity to surface related phenomena revealing the SPR as expected at $3.15 \mathrm{eV}$. The spatial distribution of the energy loss intensity at these,(Fig 1c\&d) reveals characteristic distribution for the bulk and surface plasmons as series of "energy filtered" images obtained by constant energy cuts through the $3 \mathrm{~d}$ spectral image data cube.

The plasmons of a rod-shaped nanoparticle can be categorized into the longitudinal and transverse modes where the direction of the collective electronic oscillations is parallel and perpendicular to the rod axis, respectively. The longitudinal modes can be described as 
resonant modes of an antenna that are described by the wire's length. Higher order transverse plasmons unlike their longitudinal counterpart, show little frequency dependence. Figure 2 displays energy-filtered images of the first 3 longitudinal modes (a) of a 30x300nm silver nanowire at 0.95,1.5 and 2.2 eV's respectively. The transverse (b) and bulk(c) modes are similar to the ones observed for the spherical nanoparticle. The wavelength dependence of the modes energy, the silver plasmon dispersion relation, can be derived by measuring the wave number from the distinct modes and the associated mode energies (see figure $2 \mathrm{~d}$ ).

In summary, the unmatched lateral resolution modern microscopes combined with the high energy resolution of and spectrometers is a unique tool to understand optical properties of metallic nanostructures.

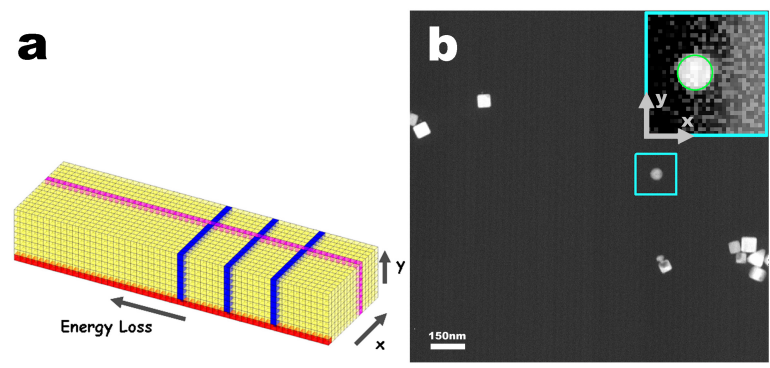

C

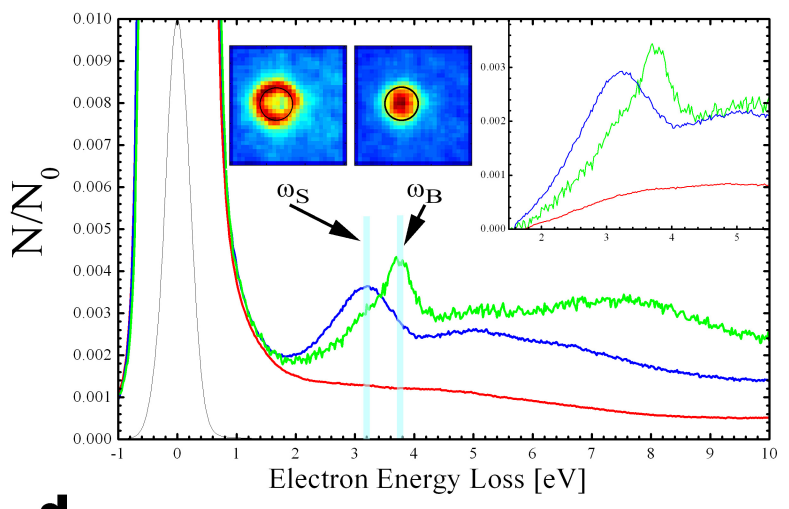

d
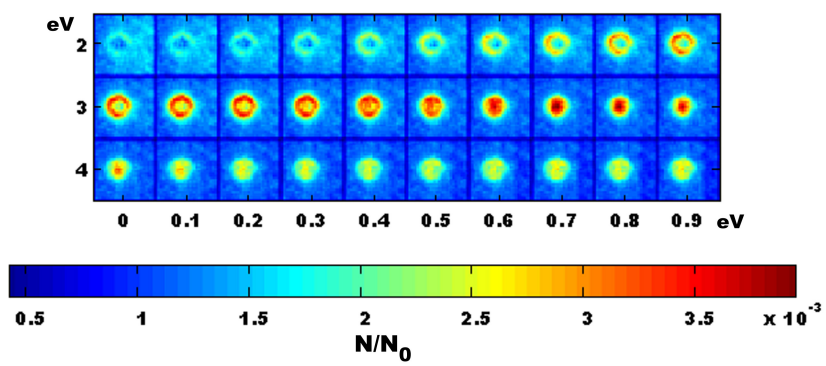

Figure 1. Spectral image of a spherical silver nanoparticle. Schematic description of the 3D EELS spectral image (a) and a high angle annular dark field image of the sample (b). The blue square indicates the area of the spectral image map around a spherical particle. (c) displays individual spectra representative of the the particle's center (green)and edge (blue) as well as the supporting film (red).In the insert spectra after subtraction of the ZLP. The left upper corner shows the spectra after subtraction of the ZLP. (d) spatial distribution of the energy loss signal as a function of energy in the 2 to $4 \mathrm{eV}$ range.
References

[1] L.M. Liz-Marzan, Langmuir 22 (2006) 32.

[2] Y. Yin, A. P. Alivisatos, Nature 437 (2005) 664.

[3] This work was performed at the Molecular Foundry and National Center for Electron Microscopy, Lawrence Berkeley National Laboratory, and was supported by the Office of Science, Office of Basic Energy Sciences, of the U.S. Department of Energy under Contract No. DE-AC02-05CH11231

a
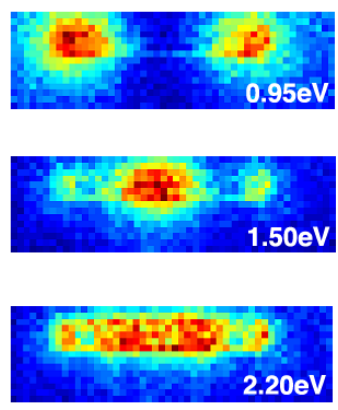

b
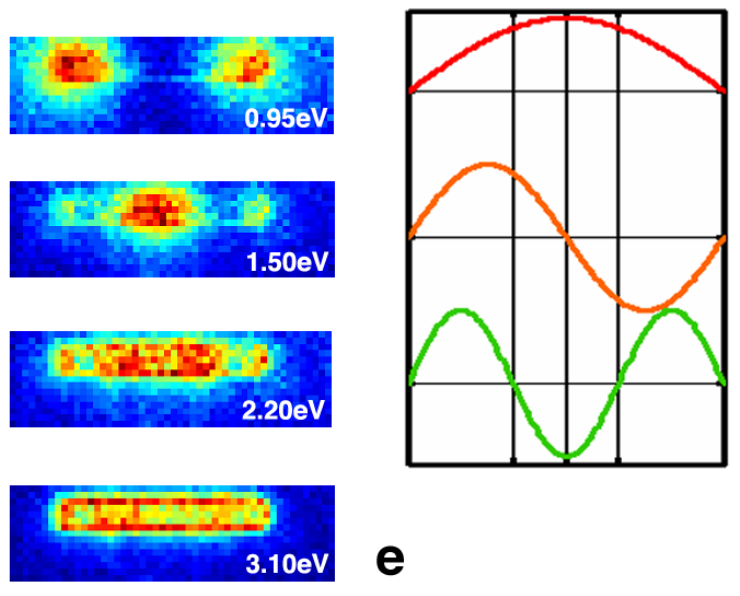

C
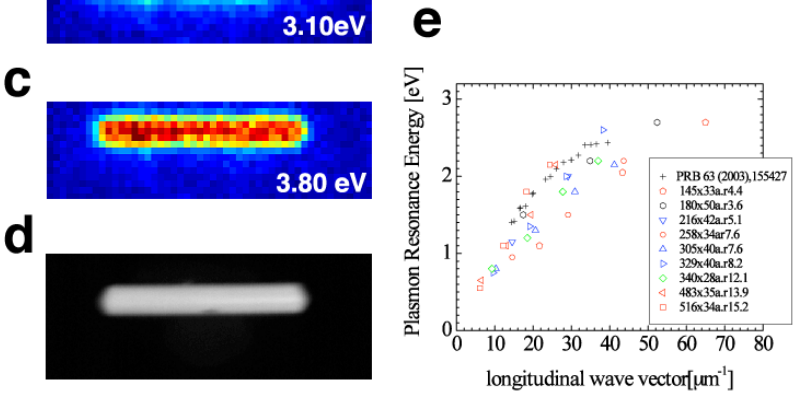

Figure2. Spectral imaging revealing the plasmonic modes of a $30 \times 300 \mathrm{~nm}$ silver nanorod. The characteristic signatures of 3 longitudinal modes (a) as well as the transverse (b) and bulk (c)plasmons can be clearly resolved. (d) The HAADF image. (e) the silver plasmon dispersion relation obtained from 9 different nanowires with aspect ratios of 3-15. 